\title{
Antifungal susceptibility profile of Candida clinical isolates from 22 hospitals of São Paulo State, Brazil
}

\author{
D.K.B. Rodrigues ${ }^{1 \oplus \bowtie}$, L.X. Bonfietti ${ }^{1 \oplus}$, R.A. Garcia ${ }^{1 \oplus}$, M.R. Araujo $^{1 \oplus}$, J.S. Rodrigues ${ }^{1 \oplus}$ \\ V.M.F. Gimenes ${ }^{20}$, and M.S.C. Melhem ${ }^{1,30}$ \\ ${ }^{1}$ Núcleo de Micologia do Instituto Adolfo Lutz, Secretaria de Saúde, São Paulo, SP, Brasil \\ ${ }^{2}$ Laboratório de Micologia Médica-LIM 53, Instituto de Medicina Tropical, Universidade de São Paulo, São Paulo, SP, Brasil \\ ${ }^{3}$ Escola de Medicina, Universidade Federal do Mato Grosso do Sul, Mato Grosso do Sul, MS, Brasil
}

\begin{abstract}
This study aimed to evaluate the frequency of cryptic Candida species from candidemia cases in 22 public hospitals in São Paulo State, Brazil, and their antifungal susceptibility profiles. During 2017 and 2018, 144 isolates were molecularly identified as 14 species; C. parapsilosis (32.6\%), C. albicans (27.7\%), C. tropicalis (14.6\%), C. glabrata (9.7\%), C. krusei (2.8\%), C. orthopsilosis (2.8\%), C. haemulonii var. vulnera (2.1\%), C. haemulonii (1.4\%), C. metapsilosis (1.4\%), C. dubliniensis (1.4\%), C. guilliermondii (1.4\%), C. duobushaemulonii (0.7\%), C. kefyr (0.7\%), and C. pelliculosa (0.7\%). Poor susceptibility to fluconazole was identified in $6.4 \%$ of $C$. parapsilosis isolates $(0.12$ to $>64 \mu \mathrm{g} / \mathrm{mL}), 50 \%$ of $C$. guilliermondii $(64 \mu \mathrm{g} / \mathrm{mL}), 66.6 \%$ of C. haemulonii var. vulnera $(16-32 \mu \mathrm{g} / \mathrm{mL}$ ), and C. duobushaemulonii strain (MIC $64 \mu \mathrm{g} / \mathrm{mL}$ ). Our results corroborated the emergence of $C$. glabrata in Brazilian cases of candidemia as previously reported. Importantly, we observed a large proportion of non-wild type $C$. glabrata isolates to voriconazole $(28.6 \% ;<0.015$ to $4 \mu \mathrm{g} / \mathrm{mL})$ all of which were also resistant to fluconazole (28.6\%). Of note, C. haemulonii, a multidrug resistant species, has emerged in the Southeast region of Brazil. Our findings suggested a possible epidemiologic change in the region with an increase in fluconazole-resistant species causing candidemia. We stress the relevance of routine accurate identification to properly manage therapy and monitor epidemiologic trends.
\end{abstract}

Key words: Candidemia; Drug resistance; Candida haemulonii; Candida glabrata; Echinocandins

\section{Introduction}

Candida is a genus of yeasts that most frequently cause fungal bloodstream infections, known as candidemia, widely diffused in intensive care units, characterizing a life-threatening condition in critically ill patients (1). Candidemia cases are rising and candidemia is becoming a public health problem with a high financial burden, especially in developing countries (1).

The epidemiology of candidemia may vary according to geography, patient characteristics, and hospital system. Some risk factors associated with candidemia are the use of immunosuppressive drugs, antibiotics, and central venous catheters, especially in patients with hematologic diseases, neutropenia, and diabetes mellitus (2). In Brazil, the mortality rate of candidemia cases can be over $50 \%$, higher than countries in Europe and North America (3).

Although $C$. albicans is the predominant species, studies have noted an increase of non-albicans Candida species. Molecular identification tools allow the identification of new cryptic species within larger species complexes, e.g., C. albicans, C. parapsilosis, C. glabrata, C. haemulonii, and Meyerozyma guilliermondii (Candida guilliermondii) (4). Understanding the distribution of cryptic species can help in the clinical management because some species show reduced susceptibility to triazoles or echinocandins (1).

Due to the large size, variable climate, and socialeconomical variability of regions in Brazil, there are relatively few representative data describing the etiologic agents of candidemia down to cryptic species in Brazil, or their susceptibility to antifungal drugs. We add new information on the cryptic species distribution and resistance occurrence among Brazilian isolates causing candidemia.

\section{Material and Methods}

We collected 144 yeast isolates from individual patients that were submitted to the Reference State Laboratory Institute Adolfo Lutz between 2017 and 2018, from 22 public hospitals, including tertiary general hospitals (54.5\%), teaching hospitals $(40.9 \%)$, and infectious diseases hospitals 
(4.5\%) distributed across 14 cities in São Paulo State, Brazil, as part of the National Antimicrobial Resistance Surveillance. The isolates were initially recovered from glycerol stock at $-20^{\circ} \mathrm{C}$ and were subcultured onto CHROMagar $^{\mathrm{TM}}$ Candida medium (Difco, USA) to evaluate colony purity and viability. Standard phenotypic methods such as microscopic morphology on corn meal agar plus Tween 80 , growth at $37^{\circ} \mathrm{C}$ and $42^{\circ} \mathrm{C}$, assessment of growth on 19 carbon and 2 nitrogen sources, fermentation of six carbohydrates, and morphological features were used to initially assign species (5).

\section{Molecular identification}

For molecular identification of isolates initially identified as C. albicans, C. glabrata, and C. parapsilosis, we used PCR (polymerase chain reaction), multiplex PCR, and PCR-RFLP (polymerase chain reaction-restriction fragment length), respectively, with specific primers following previously published protocols (6-8). The DNA extraction was performed as described by Green and Sambrook (9) and concentration and purity were checked using NanoDrop 1000 (Thermo Fisher Scientific, USA). Additionally, matrix-assisted laser desorption/ionizationtime of flight mass spectrometry (MALDI-TOF MS]-based identification of $C$. haemulonii and $C$. guilliermondii species complex isolates was performed using the ethanol/formic acid extraction protocol provided by the manufacturer (Bruker Daltonics, USA).

\section{Antifungal susceptibility testing}

The minimum inhibitory concentrations (MICs) of antifungal agents for all of the isolates were determined by the reference broth microdilution method according to Clinical and Laboratory Standards Institute (CLSI) M27-ed4 (10) for fluconazole (FCZ), voriconazole (VCZ), caspofungin (CAS), anidulafungin (AND), micafungin (MICA), and amphotericin B (AmB). As a quality control, C. parapsilosis ATCC 22019 and C. krusei ATCC 6258 were used. MIC breakpoints were interpreted according to CLSI document M60 (11). When breakpoints were not available, the epidemiological cutoff values (ECVs) were employed to classify the isolates $(12,13)$ (Supplementary Table S1). The ECVs allowed the differentiation between wild type and non-wild type isolates. Some species remain without breakpoints or ECVs for antifungals.

\section{Results}

The 144 non-duplicate isolates were obtained from hemocultures coming from public hospitals that included regular hospitals $(54.5 \%)$, teaching hospitals $(40.9 \%)$, and hospitals specializing in infectious diseases (4.5\%) located in 14 cities in São Paulo State, the most populous state in Brazil. The C. parapsilosis complex represented $36.8 \%(n=53)$ of the total species, of which C. parapsilosis sensu stricto (s.s.) accounted for $88.7 \% \quad(n=47)$,
C. orthopsilosis $7.5 \%(n=4)$, and C. metapsilosis $3.8 \%$ $(n=2)$. The C. albicans complex was the second most frequent, representing $29.1 \%(n=42)$ of the total species with the following species C. albicans s.s. $95.2 \%(n=40)$ and C. dubliniensis $4.8 \% \quad(n=2)$. No cryptic species were found for the C. grabrata complex, so the species C. glabrata s.s. was found with a frequency of $9.7 \%$ $(n=14)$. The C. haemulonii complex represented $4.2 \%$ $(n=6)$ of the candidemia cases. Within the complex, the following frequencies were found: $C$. haemulonii s.s. $33.3 \%(n=2)$, C. haemulonii var. vulnera $50 \%(n=3)$, and C. duobushaemulonii $16.66 \%(n=1)$. The frequency for C. guilliermondii s.s. was $1.2 \%(n=2)$ and no cryptic species were found. The frequencies of other species not belonging to a species complex were: C. tropicalis $14.6 \%(n=21)$, C. krusei $2.8 \%(n=4)$, C. kefyr $0.7 \%(n=1)$, and $C$. pelliculosa $0.7 \%(n=1)$.

The $\mathrm{MIC}_{50}$ and MIC range of the susceptibility testing are summarized in Supplementary Table S2. Most of the isolates were susceptible or wild type to the tested antifungals. For amphotericin B, high MICs for C. duobushaemulonii were encountered. We found resistance or nonwild type to fluconazole (FCZ) in C. parapsilosis s.s. (6.4\%; $3 / 47)$, C. glabrata s.s. (28.6\%; 4/14), C. guilliermondii s.s. $(50 \% ; 1 / 2)$, and high MICs to C. haemulonii var. vulnera $(100 \% ; 3 / 3)$ and for the single strain of C. duobushaemulonii. For voriconazole (VCZ), resistance or non-wild type strains were observed among C. krusei (25\%; 1/4), C. guilliermondii s.s. (50\%; 1/2), and C. glabrata s.s. $(28.6 \% ; 4 / 14)$ isolates. Four strains of C. glabrata s.s. $(28.6 \% ; 4 / 14)$, one of $C$. tropicalis $(4.8 \% ; 1 / 21)$, and one of C. guilliermondii s.s. $(50 \% ; 1 / 2)$ exhibited high MICs for both FCZ and VCZ. The only strain of $C$. kefyr was non-wild type for CAS and VCZ and one isolate of C. dubliniensis was non-wild type for CAS. The percentage of resistant or non-wild type strains to fluconazole and voriconazole are summarized in Figure 1.

\section{Discussion}

Antifungal susceptibility testing and the use of molecular biology are important tools not only as a subsidy for therapeutic guidance but also for monitoring the trend in the occurrence of species and resistance to antifungal agents over time in a given region as an epidemiological surveillance tool. Several studies that have focused on candidemia demonstrate new resistant and emerging species $(14,15)$. Brazil is one of the most populated countries in the world and presents a remarkable variety of regions in distinct stages of development. São Paulo is the most developed state and has the highest number of hospitals $(>800)$ in the country, but few data on the causative agents of candidemia are available $(3,16-18)$. The comprehensive regional distribution of causative species and antifungal susceptibility patterns provides support to surveillance programs at both the local and 


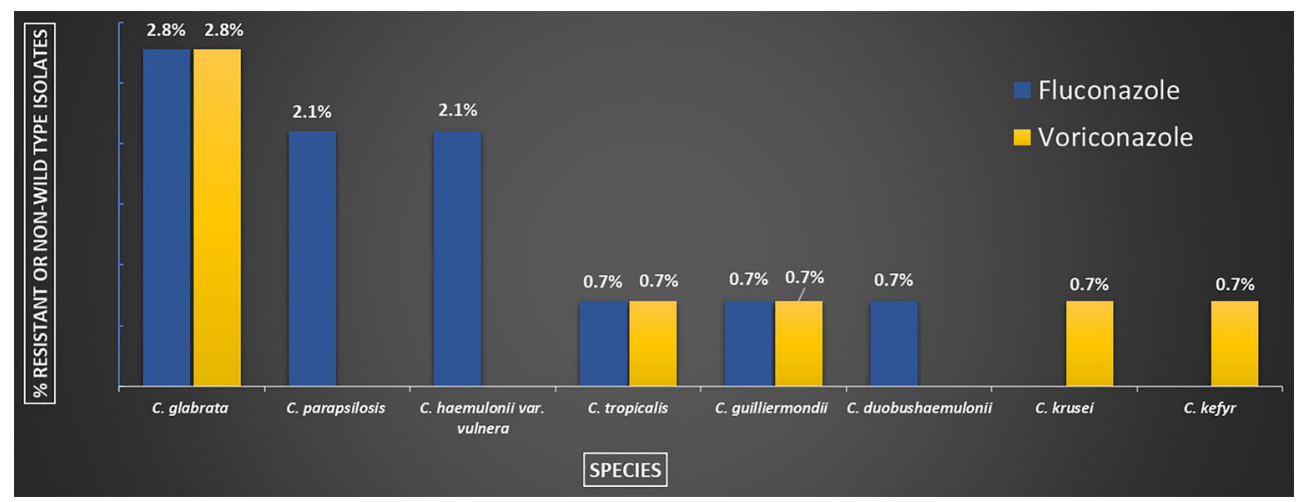

Figure 1. Percentage of fluconazole $(n=13)$ and voriconazole $(n=8)$ resistant, non-wild type or with high minimum inhibitory concentrations for 144 bloodstream Candida isolates, from hospitals in São Paulo, Brazil (2017-2018).

national levels and has a positive impact on patient survival (19).

The relative distribution of species causing candidemia can vary according to hospital characteristics (20). In this study, we tested isolates obtained from public hospitals, locally identified as non-C. albicans, and although there are some limitations in diagnosis due to deficiency of resources in these hospitals (21), in such settings, a large proportion $(40.9 \%)$ were teaching hospitals.

We verified $C$. parapsilosis s.s. as the prevalent species $(31.9 \%)$, followed by $C$. albicans $(27.8 \%)$. The relevance of $C$. parapsilosis s.s. in Brazilian candidemia cases has been demonstrated in previous studies $(22,23)$ and seems to be associated with cases identified at public hospitals (24).

Candida glabrata was previously recognized as an emerging species in some Brazilian health centers $(17,18,21,24)$. We also noted this trend in our isolates from the Southeast part of Brazil with C. glabrata ranking fourth, accounting for $9.7 \%$ of candidemia cases. In the Brazilian literature, few published studies have C. glabrata prevalence rates as high as $8 \%(18,24-27)$. Candida glabrata is recognized as an emerging species in Brazil, similar to what has been reported in some countries in Europe, Asia, and South America while it has already emerged in the United States as the second most prevalent species (28-31). The percentage of VCZ nonwild type C. glabrata isolates was high $(28.6 \%)$ in our study. These isolates were often FCZ-resistant as well, one of the highest rates among Brazilian studies $(22,24$, 32). We observed no resistance for echinocandins in C. glabrata. Only $\sim 50 \%$ of Latin American hospitals have echinocandins available for candidemia treatment, thus azoles resistance characterizes a serious health problem (33).

The frequency of $C$. guilliermondii s.s $(2 \%)$ verified in this study is similar to what has been previously reported in Brazil $(18,20)$. The largest candidemia study performed in South and Central America showed a $6.5 \%$ prevalence of $C$. guilliermondii s.s on the continent and an incredibly high burden $(20.7 \%)$ in Honduras (26). There was one FCZ- and VCZ-resistant non-wild type C. guilliermondii s.s strain but the strain and the other isolate were susceptible or wild type to all other antifungals tested. As previously described in Brazil, our C. guilliermondii s.s and C. glabrata isolates were more susceptible to FCZ than reported in other countries $(20,34)$.

The relative rate of $C$. haemulonii complex isolates was high $(4.2 \%)$ compared to previous studies showing frequencies $<2 \%$ in Brazil $(35,36)$, Asia $(14,37)$, South America (26), and several countries as published by SENTRY antifungal surveillance (15). A higher frequency $(15 \%)$ of $C$. haemulonii complex was reported only in India (38), but there was no mention of usage of molecular tools essential for ascertaining the frequency of cryptic species. In our study, we did not find high MICs for $C$. haemulonii s.s., however all $C$. haemulonii var. vulnera showed elevated FCZ MICs. Candida duobushaemulonii was unique in that it showed high MICs for AmB, and in an earlier report in Sao Paulo State $(35,36)$, this species was cited as having the highest MIC values to polyenes in the complex as well as presenting elevated MICs to fluconazole (36). The lack of clinical breakpoints for this species limits the ability to characterize resistance, however, C. duobushaemulonii has a large potential to multidrug resistance. VCZ MICs were not elevated, which differs from other studies $(14,39)$. A recent Brazilian study has shown that resistance to oxidative stress along with changes in homeostasis, mitochondrial function, and ergosterol may be related to $A m B$ resistance in the C. haemulonii complex (40). Cases of C. haemulonii complex are increasing according to recent reports, and more research is needed to understand antifungal susceptibility within the complex (36). C. kefyr is a rare cause of candidemia $(>1 \%)$, and the single isolate in our study was non-wild type for both VCZ and CAS.

This study has some limitations. All bloodstream isolates are expected, but not compulsory, to be sent to 
the State Reference Laboratory, but some Candida isolates from the hospitals under surveillance may have been left out of the study. Our study covered isolates from 22 hospitals and, although it encompassed a great variety of hospitals, it may not be representative of the entire country. Even if this study comprises only a fraction of the large geographic and demographic territory of Brazil, our findings may still add useful etiologic data to the global surveillance of candidemia.

We showed that although C. albicans s.s. and C. parapsilosis s.s. remain the most frequent species in São Paulo state, C. haemulonii complex was verified to be a frequent causative agent of candidemia in the state. Moreover, as C. haemulonii complex is easily misidentified as $C$. auris, a multidrug-resistant and emerging species, accurate characterization of candidemia species is justified. Additionally, we highlight the emergence of C. glabrata in Brazil and, notably, the significant number of strains associated with high MICs to both FCZ and VCZ. The multidrug-resistant species of $C$. guilliermondii complex are still rare in our region.

Finally, many common Candida species are now considered species complexes and the identification of cryptic species is problematic but of clinical relevance since they

\section{References}

1. Pfaller MA, Castanheira M. Nosocomial candidiasis: antifungal stewardship and the importance of rapid diagnosis. Med Mycol 2016; 54: 1-22, doi: 10.1093/mmy/myv076.

2. Pappas PG, Lionakis MS, Arendrup MC, Ostrosky-Zeichner L, Kullberg BJ. Invasive candidiasis. Nat Rev Dis Prim 2018; 4: 18026, doi: 10.1038/nrdp.2018.26.

3. Canela HMS, Cardoso B, Vitali LH, Coelho HC. Prevalence, virulence factors and antifungal susceptibility of Candida spp. isolated from bloodstream infections in a tertiary care hospital in Brazil. Mycoses 2018; 61: 11-21, doi: 10.1111/ myc. 12695.

4. Cletus Kurtzman, Boekhout FT. The Yeasts: a taxonomic study. 2011. p 2354.

5. Larone DH. Medically important fungi: a guide to identification-5 th Edition. Vol. 5, Laboratory Medicine; 2014.

6. Romeo O, Criseo G. First molecular method for discriminating between Candida africana, Candida albicans, and Candida dubliniensis by using hwp1 gene. Diagn Microbiol Infect Dis 2008; 62: 230-233, doi: 10.1016/j.diagmicrobio. 2008.05.014.

7. Luo G, Mitchell TG. Rapid identification of pathogenic fungi directly from cultures by using multiplex PCR. J Clin Microbiol 2002; 40: 2860-2865, doi: 10.1128/JCM.40.8. 2860-2865.2002.

8. Tavanti A, Davidson AD, Gow NAR, Maiden MCJ, Odds FC. Candida parapsilosis Groups II and III. J Clin Microbiol 2005; 43: 284-292, doi: 10.1128/JCM.43.1.284.

9. Green MR, Sambrook J. Molecular cloning: a laboratory manual. Vol. 33. 2012. p 362-375.

10. Clinical and Laboratory Standards Institute. M27. Reference method for broth dilution antifungal susceptibility testing of may have different infection sites, infection severity, and distinct antifungal susceptibility patterns. Although there is no consensus about the need to routinely differentiate sibling species in the clinical laboratory, strategies such as a laboratory network for centralizing molecular testing in big reference centers, as occurs in Brazil, contributes significantly to describing epidemiological data such as species distribution and antifungal susceptibility profile in geographical regions.

\section{Supplementary Material}

Click to view [pdf].

\section{Acknowledgments}

The authors gratefully acknowledge Dr. Shawn R. Lockhart who read the manuscript and provided critical comments. We appreciate the technical work from Andrés Avelino Baez. This study was supported by fellowships from the São Paulo Research Foundation, FAPESP, São Paulo, Brazil (Grants \#2018/18996-9 and \#2017/ 50333-7) and the National Council of Research, Ministry of Science and Culture, CNPq, Brasilia, Brazil. yeasts. 4th Edition. Wayne: Clinical and Laboratory Standards Institute. 2017. p 1-46, (https://clsi.org/standards/ products/microbiology/documents/m27/).

11. Clinical and Laboratory Standards Institute. M60. Performance standards for antifungal susceptibility testing of yeasts. Wayne: Clinical and Laboratory Standards Institute. 2017. p 1-12.

12. Pfaller MA, Diekema DJ. Progress in antifungal susceptibility testing of Candida spp. by use of Clinical and Laboratory Standards Institute broth microdilution methods, 2010 to 2012. J Clin Microbiol 2012; 50: 2846-2856, doi: 10.1128/ JCM.00937-12.

13. Clinical and Laboratory Standards Institute. M59. Epidemiological cutoff values for antifungal susceptibility testing Wayne: Clinical and Laboratory Standards Institute. 2018. $1-13 \mathrm{p}$.

14. Xiao M, Chen SCA, Kong F, Xu XL, Yan L, Kong HS, et al. Distribution and antifungal susceptibility of Candida species causing candidemia in China: an update from the CHIF-NET study. J Infect Dis 2020; 221: S139-S147, doi: 10.1093/ infdis/jiz573.

15. Pfaller MA, Diekema DJ, Turnidge JD, Castanheira M, Jones RN. Twenty years of the SENTRY antifungal surveillance program: results for Candida species from 1997-2016. Open Forum Infect Dis 2019; 6: S79-S94, doi: 10.1093/ofid/ofy358.

16. Marra AR, Camargo LFA, Pignatari ACC, Sukiennik T, Behar PRP, Medeiros EAS, et al. Nosocomial bloodstream infections in Brazilian hospitals: analysis of 2,563 cases from a prospective nationwide surveillance study. $J$ Clin Microbiol 2011; 49: 1866-1871, doi: 10.1128/JCM.00376-11. 
17. da Matta DA, Souza ACR, Colombo AL. Revisiting species distribution and antifungal susceptibility of Candida bloodstream isolates from Latin American Medical Centers. J Fungi 2017; 3: 24, doi: 10.3390/jof3020024.

18. Moretti ML, Trabasso P, Lyra L, Fagnani R, Resende MR, Cardoso LGO, et al. Is the incidence of candidemia caused by Candida glabrata increasing in Brazil? Five-year surveillance of Candida bloodstream infection in a university reference hospital in southeast Brazil. Med Mycol 2013; 51: 225-230, doi: 10.3109/13693786.2012.708107.

19. Bassetti M, Merelli M, Righi E, Diaz-Martin A, Rosello EM, Luzzati R, et al. Epidemiology, species distribution, antifungal susceptibility, and outcome of candidemia across five sites in Italy and Spain. J Clin Microbiol 2013; 51: 41674172, doi: 10.1128/JCM.01998-13.

20. Wille MP, Guimarães T, Furtado GHC, Colombo AL. Historical trends in the epidemiology of candidaemia: Analysis of an 11-year period in a tertiary care hospital in Brazil. Mem Inst Oswaldo Cruz 2013; 108: 288-292, doi: 10.1590/S0074-02762013000300005.

21. Colombo AL, Garnica M, Camargo LFA, Da Cunha CA, Bandeira AC, Borghi D, et al. Candida glabrata: an emerging pathogen in Brazilian tertiary care hospitals. Med Mycol 2013; 51: 38-44, doi: 10.3109/13693786.2012.698024

22. Santos ER, Dal Forno CF, Hernandez MG, Kubiça TF, Venturini TP, Chassot F, et al. Susceptibility of Candida spp. isolated from blood cultures as evaluated using the M27-A3 and NEW M27-S4 approved breakpoints. Rev Inst Med Trop Sao Paulo 2014; 56: 477-482, doi: 10.1590/S0036-46652 014000600004

23. Hinrichsen SL, Falcão É, Vilella TAS, Colombo AL, Nucci M, Moura L, et al. Candidemia in a tertiary hospital in northeastern Brazil. Rev Soc Bras Med Trop 2008; 41: 394-398, doi: 10.1590/S0037-86822008000400014.

24. Doi AM, Pignatari ACC, Edmond MB, Marra AR, Camargo LFA, Siqueira RA, et al. Epidemiology and microbiologic characterization of nosocomial Candidemia from a Brazilian National Surveillance Program. PLoS One 2016; 11: e0146909, doi: 10.1371/journal.pone.0146909.

25. Colombo AL, Nucci $M$, Park BJ, Nouér SA, ArthingtonSkaggs B, Da Matta DA, et al. Epidemiology of candidemia in Brazil: a nationwide sentinel surveillance of candidemia in eleven medical centers. J Clin Microbiol 2006; 44: 28162823, doi: 10.1128/JCM.00773-06.

26. Nucci M, Queiroz-Telles F, Alvarado-Matute T, Tirabosch IN. Epidemiology of Candidemia in Latin America: a laboratorybased survey. PLoS One 2013; 8: e59373, doi: 10.1371/ journal.pone.0059373.

27. Araújo $M$, Medeiros $P$ de, Melo $A P V$ de, Bento $A$ de $O$, Souza LBFC de, Neto $F$ de $A B$, et al. Epidemiology and prognostic factors of nosocomial candidemia in Northeast Brazil: a six-year retrospective study. PLoS One 2019; 14: e0221033, doi: 10.1371/journal.pone.0210501.

28. Kakeya H, Yamada K, Kaneko Y, Yanagihara K, Tateda K, Maesaki S, et al. National trends in the distribution of Candida species causing candidemia in Japan from 2003 to 2014: a report by the epidemiological investigation committee for human mycoses in Japan. Med Mycol J 2018; 59: E19-E22, doi: 10.3314/mmj.17-00014.
29. Lindberg $\mathrm{E}$, Hammarström $\mathrm{H}$, Ataollahy $\mathrm{N}$, Kondori $\mathrm{N}$. Species distribution and antifungal drug susceptibilities of yeasts isolated from the blood samples of patients with candidemia. Sci Rep 2019; 9: 3838, doi: 10.1038/s41598019-40280-8.

30. Tsay SV, Mu Y, Williams S, Epson E, Nadle J, Bamberg WM, et al. Burden of Candidemia in the United States, 2017. Clin Infect Dis 2020; 71: e449-e453, doi: 10.1093/cid/ ciaa193.

31. Siri L, Legarraga P, García P, González T, Rabagliati R. Cambios clínicos y epidemiológicos de candidemias en pacientes adultos desde 2000 a 2013. Rev Chilena Infectol 2017; 34: 19-26, doi: 10.4067/S0716-10182017000100003.

32. Motta AL, de Almeida GMD, de Almeida Júnior JN, Burattini MN, Rossi F. Candidemia epidemiology and susceptibility profile in the largest Brazilian teaching hospital complex. Brazilian J Infect Dis 2010; 14: 441-448, doi: 10.1016/ S1413-8670(10)70091-X.

33. Falci DR, Pasqualotto AC. Clinical mycology in Latin America and the Caribbean: a snapshot of diagnostic and therapeutic capabilities. Mycoses 2019; 62: 368-373, doi: $10.1111 /$ myc. 12890 .

34. da Matta DA, de Almeida LP, Machado AM, Azevedo AC, Kusano EJU, Travassos NF, et al. Antifungal susceptibility of 1000 Candida bloodstream isolates to 5 antifungal drugs: results of a multicenter study conducted in São Paulo, Brazil, 1995-2003. Diagn Microbiol Infect Dis 2007; 57: 399404, doi: 10.1016/j.diagmicrobio.2006.10.011.

35. de Almeida JRN, Assy JGPL, Levin AS, del Negro GMB, Giudice MC, Tringoni MP, et al. Candida haemulonii complex species, Brazil, January 2010-march 2015. Emerg Infect Dis 2016; 22: 561-563, doi: 10.3201/ eid2203.151610.

36. Lima SL, Francisco EC, Júnior JN de A, Santos DW de CL, Carlesse F, Queiroz-Telles F, et al. Increasing prevalence of multidrug-resistant Candida haemulonii species complex among all yeast cultures collected by a reference laboratory over the past 11 years. J Fungi 2020; 6: 110, doi: 10.3390/ jof6030110.

37. Tan BH, Chakrabarti A, Li RY, Patel AK, Watcharananan SP, Liu Z, et al. Incidence and species distribution of candidaemia in Asia: a laboratory-based surveillance study. Clin Microbiol Infect 2015; 21: 946-953, doi: 10.1016/j.cmi. 2015.06.010.

38. Oberoi JK, Wattal C, Goel N, Raveendran R, Datta S, Prasad K. Non-albicans Candida species in blood stream infections in a tertiary care hospital at New Delhi, India. Indian J Med Res 2012; 136: 997-1003.

39. Ramos R, Caceres DH, Perez M, Garcia N, Castillo W, Santiago E, et al. Emerging multidrug-resistant Candida duobushaemulonii infections in Panama hospitals: Importance of laboratory surveillance and accurate identification. J Clin Microbiol 2018; 56: e00371-18, doi: 10.1128/JCM. 00371-18.

40. Silva LN, Oliveira SSC, Magalhães LB, Andrade Neto VV, Torres-Santos EC, Carvalho MDC, et al. Unmasking the Amphotericin B resistance mechanisms in Candida haemuIonii species complex. ACS Infect Dis 2020; 6: 1273-1282, doi: 10.1021/acsinfecdis.0c00117. 